\title{
Zusätzliche MR-Spektroskopie im Test
}

Welchen Vorteil bietet die Kombination aus endorektaler KernspinBildgebung zusammen mit einer Kernspin-Spektroskopie? US-amerikanische Kollegen gingen dieser Frage in einer Studie mit 134 Teilnehmern nach.

Z ur Diagnostik eines Prostatakarzinoms trägt die endorektale Kernspin-Untersuchung gute Ergebnisse bei. Neu ist die Kombination aus diesem Verfahren zusammen mit einer KernspinSpektroskopie, die auf metabolischen Veränderungen in Tumorzellen basiert. Diese kombinierte Methode soll die Detektion von Tumoren in der peripheren Zone (PZ) weiter verbessern.

Das American College of Radiology Imaging Network (ACRIN) führte eine prospektive Multicenter-Studie durch, bei dem das kombinierte Verfahren mit der endorektalen MR-Bildgebung allein verglichen wurde. Von 134 Patienten mit histologisch gesichertem Adenokarzinom der Prostata, bei denen eine radikale Prostatektomie anstand, wurden T1- und T2-gewichtete MR-Sequenzen sowie eine spektroskopische Sequenz bei 1,5 Tesla durchgeführt.

Acht Untersucher beurteilten unabhängig und Sextanten-bezogen die Wahrscheinlichkeit für ein peripheres Prostatakarzinom in einer Fünf-Punkte-Skala - zunächst mit der konventionellen MRBildgebung allein und später mit dem kombinierten Verfahren. Referenz war die Anwesenheit von Karzinomgewebe in der zentralen histopathologischen Untersuchung der entfernten Prostata.
Insgesamt standen 110 komplette Datensätze zur Verfügung. Die Auswertung ergab für beide Verfahren ähnliche Werte bei Erfassung eines peripheren Prostatakarzinoms: Die Fläche unter der ROC-Kurve (AUC) lag beim Kernspinbild bei 0,60, für das kombinierte Verfahren bei 0,58. Die AUC-Werte der einzelnen Untersucher korrelierten gut miteinander.

Fazit: In dieser Studie war die Kombination aus endorektaler Kernspin-Bildgebung und -Spektroskopie bei der Detektion peripherer Prostatakarzinome der Bildgebung allein nicht überlegen. af

Weinreb JC et al. Prostate cancer: Sextant localization at MR imaging and MR spectroscopic imaging before prostatectomy results of ACRIN prospective multi-institutional clinicopathologic study.

Radiology 2009; 251: 122-33.

\section{Wichtig bei Krebspatienten: Psychische Störungen erfassen}

Wie erkennen behandelnde Kollegen am besten die Patienten, die massiv durch Angst oder Depression beeinträchtigt sind? Forscher analysierten dazu verschiedene Cut-off-Werte eines geeigneten Befragungstests.

Z ur Einschätzung betroffener Patienten bietet sich die HADS (Hospital Anxiety and Depression Scale) an. Dieser Test besteht aus 14 Statements, zu denen der Patient jeweils in vier Abstufungen Stellung nimmt. Insgesamt kann ein Maximalwert von 42 Punkten erreicht werden, ab acht Punkten besteht nach den Testautoren der Verdacht auf eine psychische Erkrankung. Darüber hinaus gibt es jeweils Subskalen für Angst und Depression. Dieser Test war allerdings für die Grundversorgung konzipiert, nicht für onkologische Patienten in der Akuttherapie.

In der vorliegenden Studie wurden 689 onkologische Patienten aus Leipziger Kliniken evaluiert. Bei allen erfolgte innerhalb der ersten drei Tage der stationären Therapie die Befragung mit dem HADS. Danach führte ein Psychologe, der über die HADS-Punktzahl nicht informiert war, ein strukturiertes Interview gemäß dem DSM-IV (Diagnostic and Statistical Manual of Mental Disorders IV) durch.

$32 \%$ der Patienten wiesen entsprechend dem DSM-IV eine psychische Störung auf. Die häufigsten Erkrankungen waren: Depressionen, Anpassungsstörungen, Phobien, generalisierte Angststörung und Alkoholabhängigkeit.

Mit einem Cut-off von $\geq 16$ Punkten konnten 59\% der Fälle erkannt werden (Sensitivität) bei einer Spezifität von $76 \%$. Bei einem HADS-Wert von $\geq 13$ lagen die Sensitivität bei $76 \%$ und die Spezifität bei $60 \%$. Ein Cut-off von $\geq 6$ bedeutete, dass $95 \%$ der Störungen entdeckt wurden, allerdings bei einer Spezifität von nur $20 \%$. Den besten Kompromiss zwischen Sensitivität und Spezifität sahen die Autoren beim Cut-offWert von $\geq 13$ Punkten. Für den Einsatz in der Klinik favorisierten sie jedoch den niedrigeren Wert von $\geq 6$ Punkten.

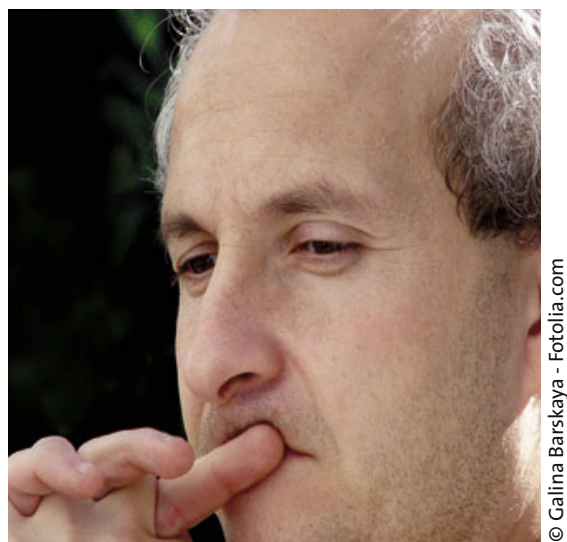

Nicht immer ist eine Depression bei Krebspatienten auf den ersten Blick zu erkennen.

Fazit: Rund ein Drittel der Krebspatienten zeigte in der vorliegenden Studie zu Beginn der Akutherapie eine psychische Störung. Mit Hilfe des HADS ist eine einfache Evaluierung von Patienten möglich. Für die klinische Praxis empfehlen die deutschen und englischen Autoren einen Cut-off-Wert von $\geq 6$ Punkten auf dieser Skala. af

Singer S et al. Hospital anxiety and depression scale cutoff scores for cancer patients in acute care. Br J Cancer 2009; 100: 908-12. 